\title{
Multifunction waveform generator for EM receiver testing
}

\author{
Kai Chen, Sheng Jin, and Ming Deng \\ School of Geophysics and Information Technology, China University of Geosciences, Beijing, China
}

Correspondence: Kai Chen (ck@cugb.edu.cn)

Received: 29 July 2017 - Discussion started: 22 August 2017

Revised: 7 December 2017 - Accepted: 15 December 2017 - Published: 29 January 2018

\begin{abstract}
In many electromagnetic (EM) methods - such as magnetotelluric, spectral-induced polarization (SIP), timedomain-induced polarization (TDIP), and controlled-source audio magnetotelluric (CSAMT) methods - it is important to evaluate and test the EM receivers during their development stage. To assess the performance of the developed EM receivers, controlled synthetic data that simulate the observed signals in different modes are required. In CSAMT and SIP mode testing, the waveform generator should use the GPS time as the reference for repeating schedule. Based on our testing, the frequency range, frequency precision, and time synchronization of the currently available function waveform generators on the market are deficient. This paper presents a multifunction waveform generator with three waveforms: (1) a wideband, low-noise electromagnetic field signal to be used for magnetotelluric, audio-magnetotelluric, and long-period magnetotelluric studies; (2) a repeating frequency sweep square waveform for CSAMT and SIP studies; and (3) a "positive-zero-negative-zero" signal that contains primary and secondary fields for TDIP studies. In this paper, we provide the principles of the above three waveforms along with a hardware design for the generator. Furthermore, testing of the EM receiver was conducted with the waveform generator, and the results of the experiment were compared with those calculated from the simulation and theory in the frequency band of interest.
\end{abstract}

\section{Introduction}

Electromagnetic (EM) methods are successfully used in a variety of applications, including metal ore investigations, groundwater explorations, hydrocarbon prospecting, volcano research, and deep-earth research (Wei et al., 2010; Key, 2003). In these applications, EM methods are used to mea- sure natural- or controlled-source signals and then to interpret the underground electrical structure using data processing and inversion techniques (Osinowo and Olayinka, 2012; Scheuermann, 2016). There are many EM methods in use today; however, in this paper we are interested in magnetotelluric (MT; Cagniard, 1953), controlled-source audio magnetotelluric (CSAMT; Sandberg and Hohmann, 1982), spectralinduced polarization (SIP; Johnson, 1984), and time-domaininduced polarization (TDIP; Marshall and Madden, 1959) methods. The requirements for field instruments supporting these methods include high resolution, large exploration depth, low cost, and high-efficiency field data acquisition. All of the above EM methods are dependent on the quality of the field data acquired by the EM instrument. Therefore, the specifications of the EM receiver are of particular importance for high-quality EM prospecting. The current EM receivers - such as the V8 receiver from Phoenix Geophysics (PhoenixGeophysics, 2017), GDP32 from Zonge (Zonge, 2017), ADU-07e from Metronix (Metronix, 2017), and KMS-820 from KMS (KMS, 2017) - are all specified as being multifunction, multi-channel, and easy to use with low noise levels and few clock drift errors.

EMR6 is a new multifunctional EM receiver for deepmetal-mineral-exploration EM survey, which was developed by the China University of Geosciences (Beijing) (CUGB) and which supports the audio magnetotelluric (AMT), MT, CSAMT, SIP, and TDIP methods on the surface and in tunnels. In addition, the MT function includes the audio magnetotelluric (AMT), magnetotelluric, and long-period magnetotelluric (LMT) methods in different frequency ranges. During the development of the instrument, the receiver was tested with each method module after the electrical parameters were characterized. For the realization of a multifrequency EM receiver, Chen et al. (2010) developed an automatic testing system based on pseudo-random signals. For 
MT receiver performance evaluation, He et al. (2011) conducted a series process method with high efficiency. For the MT method module test (Ge et al., 2016), a pseudo-random bit sequence (PRBS) module was designed, which included a white-noise source that simulated a broadband naturalsource MT signal in the $5 \times 10^{-4}$ to $14 \mathrm{kHz}$ frequency range, which was suitable for the AMT and MT mode.

However, the lower-frequency range for the LMT test was deficient. For the CSAMT and SIP mode tests, which require a repeating frequency sweep square waveform, the test signal should be time-synchronized and repeated. There are function waveform generators available on the market, such as the Agilent 33250A, which support multiple broadband waveforms; however, the frequency precision and time synchronization error in these generators are deficient. For example, in the TDIP mode test, the arbitrary waveform function is suitable, but the time synchronization error is deficient.

In this paper, we describe a multifunction waveform generator. The details of the waveform generator, including the system design and the hardware principles, are discussed. Section 2 describes the requirement of the three types of waveforms. Next, we discuss the hardware principle design in Sect. 3. Section 4 describes the test results to confirm the function of the waveform generator. In Sect. 5, the discussion and the specification comparison table are presented.

\section{System design}

The multifunction waveform generator is designed to output three types of waveforms: white noise for MT (AMT/MT/LMT), a repeated swept square waveform for CSAMT and SIP modes, and a "positive-zero-negative-zero" (PZNZ) waveform for the TDIP mode.

The MT method consists of three branches - AMT, standardized MT, and LMT - and these branches differ in terms of the exploration depths of interest and the effective frequency ranges. Figure 1 illustrates the frequency ranges applicable to the three branches of the MT method. The highfrequency band in the AMT method is from $10 \mathrm{kHz}$ to $0.1 \mathrm{~Hz}$, which spans 5 decades; and the frequency band in the standardized MT method is from 320 to $5 \times 10^{-4} \mathrm{~Hz}$, which spans approximately 6 decades; the frequency band in the LMT method is from 1 to $1 \times 10^{-5} \mathrm{~Hz}$, which spans approximately 5 decades. A PRBS generator is the best choice for generating broadband signals (Amrani et al., 1998). The highest frequency is determined by the width of the smallest encoding, and the lowest frequency depends on the length of the PRBS. According to the three different frequency band modes, the length of the PRBS must be greater than $1 \times 10^{6}$. Therefore, we designed a PRBS with length $2^{N}-1$, where $N$ equals 24 and the length is $16 \mathrm{M}$. By changing the smallest code width of the PRBS, it was easy to meet the three MT modes operating in different frequency ranges.

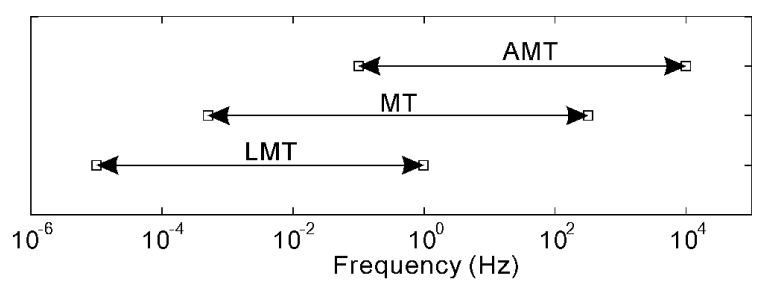

Figure 1. Three MT sounding methods with different frequency ranges.

Table 1. PRBS parameters for three MT modes.

\begin{tabular}{lcrr}
\hline Mode & Length & $\begin{array}{r}\text { Smallest code } \\
\text { width }\end{array}$ & $\begin{array}{r}\text { Available frequency } \\
\text { range }\end{array}$ \\
\hline AMT & $16 \mathrm{M}$ & $10 \mu \mathrm{s}$ & $100 \mathrm{kHz}$ to $6 \mathrm{mHz}$ \\
MT & $16 \mathrm{M}$ & $1 \mathrm{~ms}$ & $1 \mathrm{kHz}$ to $60 \mu \mathrm{Hz}$ \\
LMT & $16 \mathrm{M}$ & $100 \mathrm{~ms}$ & $10 \mathrm{~Hz}$ to $0.6 \mu \mathrm{Hz}$ \\
\hline
\end{tabular}

The formal definition of the PRBS is

$D=X^{24}+X^{7}+X^{2}+X+1$.

The length of the sequence is $16 \mathrm{M}$. The smallest code width, which changes for different fundamental frequencies, can be selected from $10 \mu \mathrm{s}, 1 \mathrm{~ms}$, and $100 \mathrm{~ms}$. Table 1 lists the parameters for the three modes.

The designed frequency range for the CSAMT method is from approximately $0.1 \mathrm{~Hz}$ to $10 \mathrm{kHz}$. The programmable frequency stepping schedule is according to the typical CSAMT frequency stepping schedule used in the field when working with the V8 receiver from Phoenix Geophysics. The frequency stepping schedule contains 41 frequency points and lasts for $50 \mathrm{~min}$. Figure 2 presents the typical frequency stepping schedule plotted on a log scale and two frequency points in a double frequency range as an approximate average on a log scale. The 41 frequency points cover 4 decades and extend from 9600 to $0.9375 \mathrm{~Hz}$. All frequency points are derived from a $12.288 \mathrm{MHz}$ high-stability clock source. To increase the lower-frequency signal-to-noise ratio (SNR), the length of the stacking time of the lower frequencies is longer than that of the higher frequencies. The longest stacking time is $323 \mathrm{~s}$ for $1.25 \mathrm{~Hz}$ in the low-frequency band, and the shortest is $40 \mathrm{~s}$ in the high-frequency band.

The designed frequency range for the SIP method is from 128 to $0.0625 \mathrm{~Hz}$. The frequency stepping schedule is the same as the typical SIP step schedule used in the field when working with the V8 receiver from Phoenix Geophysics. Figure 3 shows a typical frequency stepping schedule plotted on a log scale and frequency stepping by double. There are 12 frequency points, each of which last for $15 \mathrm{~min}$, and the 12 points cover approximately 4 decades and extend from 128 to $0.0625 \mathrm{~Hz}$. As was the case in the CSAMT mode, all frequency points could be derived from a $12.288 \mathrm{MHz}$ clock source. To increase the lower-frequency SNR, a longer stack 


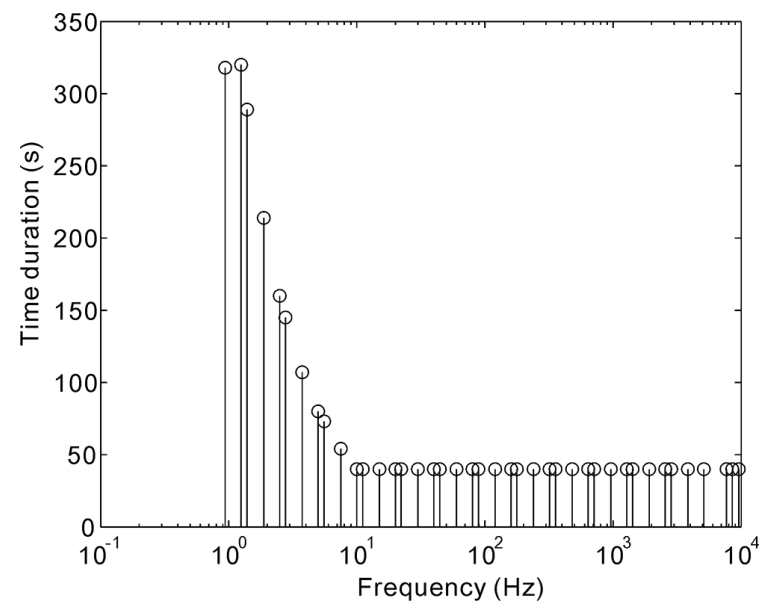

Figure 2. Stack time length for a typical CSAMT frequency stepping schedule.

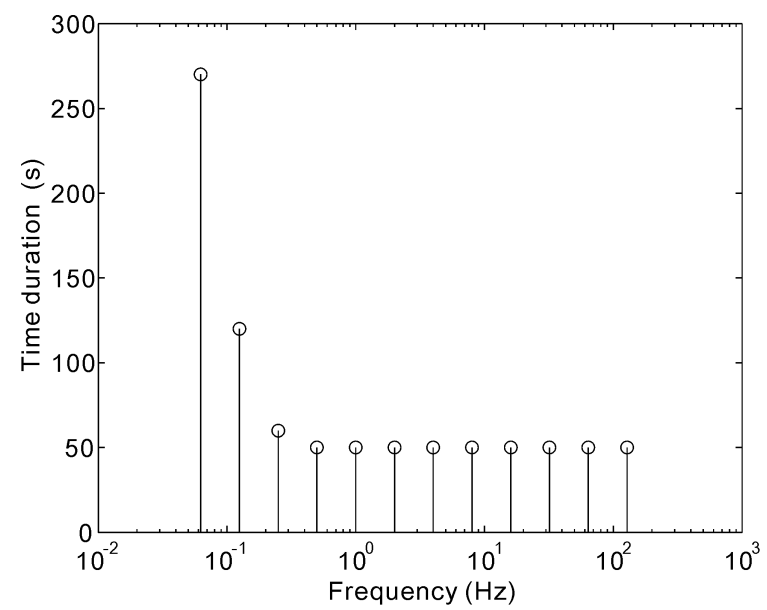

Figure 3. Stack time length for a typical SIP frequency stepping schedule.

time was used in the lower-frequency band than that used in the higher-frequency band. The longest stack time was $273 \mathrm{~s}$ in the low-frequency band at $1.25 \mathrm{~Hz}$, and each point lasted for $50 \mathrm{~s}$ in the high-frequency band.

The PZNZ waveform for the TDIP module is shown in Fig. 4 and consists of four phases: positive "on" time, "off" time, negative "on" time, and "off" time. The duty ratio is $1: 1$. In the figure, $T$ denotes the four-phase period, and the width of the pulse is $T / 4$. The term $A$ denotes the amplitude of the primary electrical field, $B$ denotes the maximum amplitude of the secondary electrical field, and $A+B$ denotes the total electrical field. The self-potential and other disturbances are not considered in the figure.

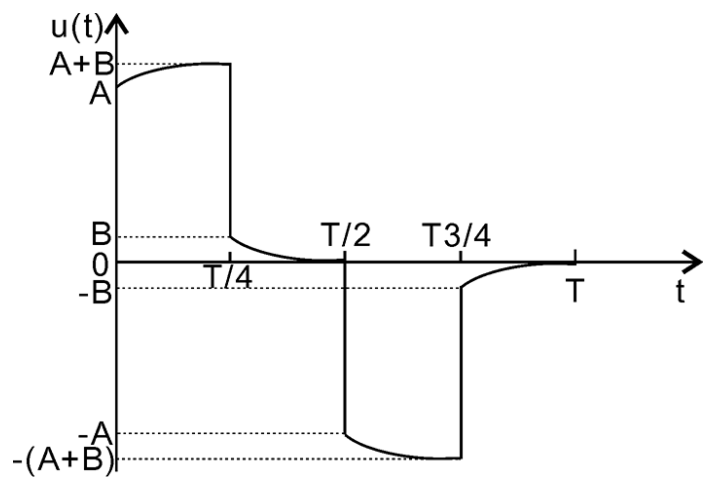

Figure 4. PZNZ waveform from a theoretical simulation.

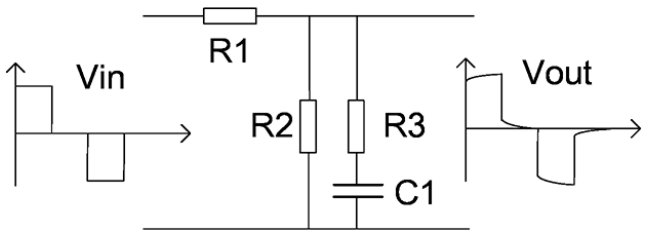

Figure 5. Schematic of the PZNZ waveform generator.

$$
\begin{aligned}
& u_{1}(t)=\left\{\begin{array}{l}
A, 0 \leq t<T / 4 \\
0, T / 4 \leq t<T / 2 \\
-A, T / 2 \leq t<3 T / 4 \\
0,3 T / 4 \leq t<T
\end{array}\right. \\
& u_{2}(t)=\left\{\begin{array}{l}
B-B e^{-t / \tau}, 0 \leq t<T / 4 \\
B e^{-\left(t-\frac{T}{4}\right) / \tau}, T / 4 \leq t<T / 2 \\
B e^{-\left(t-\frac{T}{2}\right) / \tau}-B, T / 2 \leq t<3 T / 4 \\
-B e^{-\left(t-\frac{3 T}{4}\right) / \tau}, 3 T / 4 \leq t<T
\end{array}\right.
\end{aligned}
$$

$u_{1}(\mathrm{t})$ and $u_{2}(\mathrm{t})$ denote the primary and secondary electrical fields, respectively, and the exponent attenuation curve refers to the secondary electrical field.

Figure 5 shows a schematic of the circuit used to generate the PZNZ waveform, which contains both primary and secondary electrical fields. The resistor-capacitor (RC) network transforms the PZNZ $\left(V_{\text {in }}\right)$ waveform into a PZNZ waveform $\left(V_{\text {out }}\right)$ with the secondary electrical field.

$$
\begin{aligned}
A & =\frac{R_{3} / / R_{2}}{R_{1}+R_{3} / / R_{2}} K \\
B & =\frac{R_{3} / / R_{2}}{R_{1}+R_{3} / / R_{2}} K-\frac{R_{2}}{R_{1}+R_{2}} K \\
\tau & =R_{3} C_{1}
\end{aligned}
$$

The amplitude of Vin is $\pm K$. $A$ and $B$ used in Eqs. (2) and (3) can be calculated from Eqs. (4) and (5). The time constant $(\tau)$ is given by Eq. (6). 


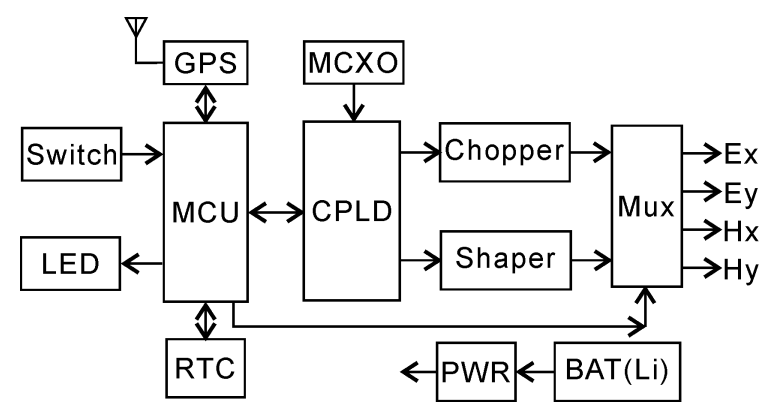

Figure 6. Block diagram of the multifunction waveform generator circuit.

\section{Hardware principle}

\subsection{Block diagram}

Figure 6 presents a block diagram of the waveform generator, which has the advantages of automation, ease of use, high phase precision, and low power. As shown in the figure, the hardware consists of an input switch, microcontroller unit (MCU), GPS module, complex programmable logic device (CPLD), compensated microprocessor crystal (MCXO), real-time clock (RTC), chopper, shaper, multiplexer, power conversion circuit, and built-in Li-ion battery package. DS3231 from Maxim Integrated is a low-cost, extremely accurate RTC with an integrated temperature-compensated crystal oscillator (TCXO) and crystal. It has the advantages of high integration level, low power, and ease of use. The low-power 8 bit microcontroller MSP430G2553 from Texas Instruments was used as the MCU, and 5M80ZE64 from Altera was used as the CPLD. The input switch is used to change the work mode without configuring the complicated external parameters. The LED is used to indicate the working status. The different work modes output different LED flash patterns. The GPS module is LEA-6T from u-blox, which provides a high-precision time pulse per second with low power consumption. The MCXO has the specifications of high-stability clock source $(12.288 \mathrm{MHz} ; \pm 30 \mathrm{ppb})$ and low power consumption ( $3.3 \mathrm{~V}$ and $12 \mathrm{~mA}$ ). To lock the GPS, the MCU receives the time information from the GPS module and writes to the RTC. The CPLD is used to implement a frequency divider, logic operator, the pulse per second (PPS) lock, and tracking. The RTC is the time counter used for circulation of the frequency stepping schedule. The chopper circuit chops a high-precision DC reference into a bipolar square waveform under the control of the CPLD. The shaper generates the TDIP PZNZ waveform. Moreover, the sum of the primary and secondary field signals is also provided as output. The multiplexer is controlled by the MCU to select either the chopper or the shaper output. The power module converts the Li-ion battery $(11.1 \mathrm{~V}$ and $10 \mathrm{Ahr})$ voltage to digital power at $3.3 \mathrm{~V}$ and analog power at $\pm 3 \mathrm{~V}$.
When the MT mode is selected, the MCU controls the CPLD to generate two independent PRBSs. The outputs $E_{x}$ and $H_{y}$ share one PRBS, whereas $E_{y}$ and $H_{x}$ share another PRBS. The two PRBSs are different for different phases. The chopper circuit converts the $3.3 \mathrm{~V}$ complementary metaloxide semiconductor (CMOS) PRBS into a bipolar $\pm 10 \mathrm{mV}$ square wave for $E_{x}$ and $E_{y}$. The amplitude of $H_{x}$ and $H_{y}$ is $\pm 100 \mathrm{mV}$. The MCU uses the multiplexer output to select the chopper output. Based on the different modes (AMT, MT, LMT), the output selected by the input switch and the smallest code width of the PRBS is changed. To decrease the power consumption, the GPS module is powered down during the MT mode.

When the CSAMT mode is selected, the MCU reads the GPS time information and writes to the RTC. The MCU controls the CPLD divisionde factor according to the frequency stepping schedule. The CPLD divides the clock source $(12.288 \mathrm{MHz})$ to the target frequency step by step. The divider is trigged by the PPS from the GPS module. The 41-frequency-point clock signal drives the chopper circuit to generate a bipolar square waveform. All frequency stepping schedules start from the reference time base of 00:00:00 and repeat from high frequency to low frequency. For example, after the power is turned on and the GPS is locked, the current time is $02: 20: 00$, the period of frequency stepping schedule is $50 \mathrm{~min}$, the residual time is $40 \mathrm{~min}$, the first output signal is frequency point no. $40(1.25 \mathrm{~Hz})$, and the output is the rest $10 \mathrm{~min}$ of the current schedule. The frequency stepping schedule is repeated continually.

The procedure in the SIP mode is the same as that described above for the CSAMT module for the step schedule with time synchronization. A typical frequency stepping schedule period is $15 \mathrm{~min}$ and contains 12 frequency points.

When the TDIP mode is selected, the RTC time is locked to the GPS module and the CPLD generates a pulse with $2 \mathrm{~s}$ width synchronization as the PPS from the GPS module to drive the shaper circuit. The reference time base is 00:00:00, and the typical period is $8 \mathrm{~s}$ with a duty ratio of $1: 1$.

To lock the GPS time to the local RTC, the MCU obtains the time information from the GPS module and writes it to the RTC. In the CSAMT, SIP, and TDIP modes, the MCU must know the current time before it can switch the repeating schedule.

\subsection{Clock source}

In accordance with the high-precision phase requirement from the CSAMT and SIP modes, an integrated u-blox GPS module and MCXO were selected as the high-stability clock source. The LEA-6T module series is a family of stand-alone GPS receivers that feature the high-performance u-blox 6 timing engine. The accuracy of the time pulse signal in the LEA-6T is approximately $30 \mathrm{~ns}$, and the time to first fix is $29 \mathrm{~s}$. The clock module is an ultra-high-stability MCXO from Vectron MX-503 with an accuracy of $\pm 30 \mathrm{ppb}$ in a temper- 


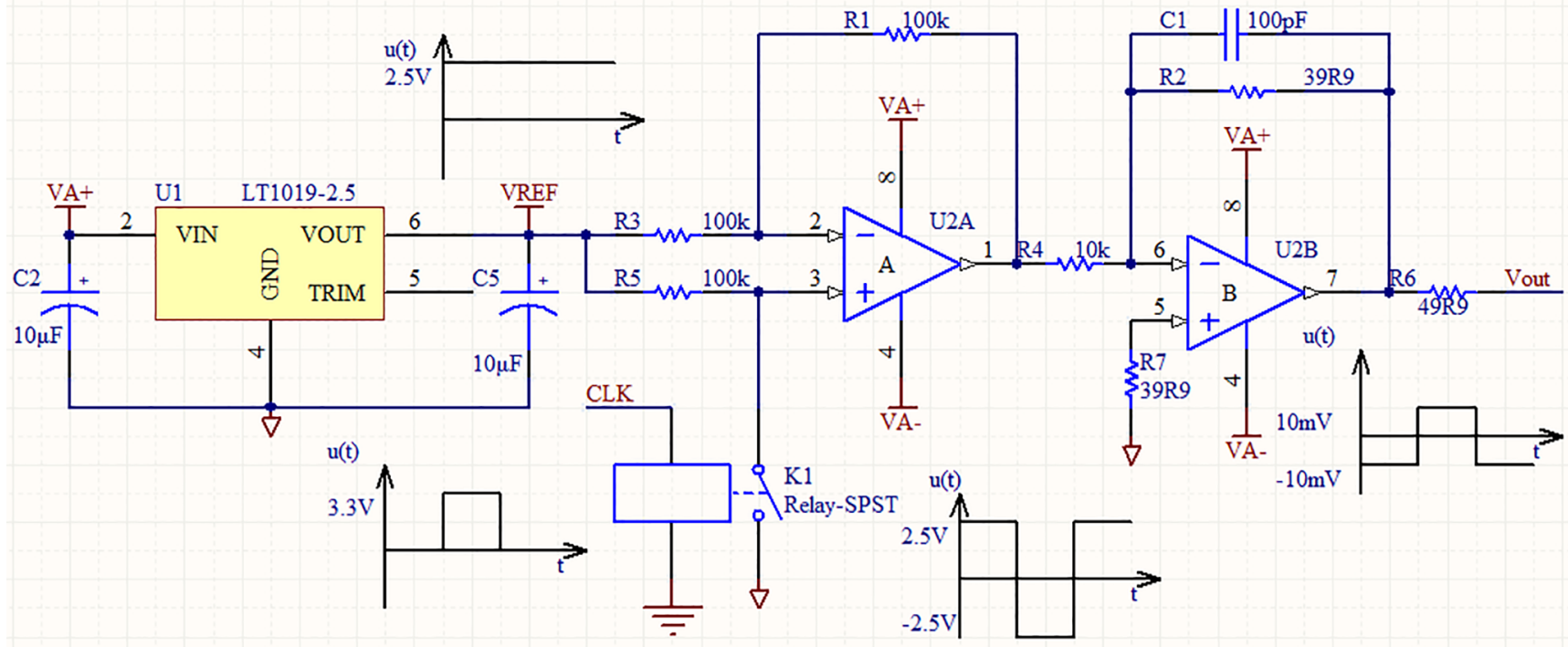

Figure 7. Schematic of the chopper circuit ( $E$ channel).

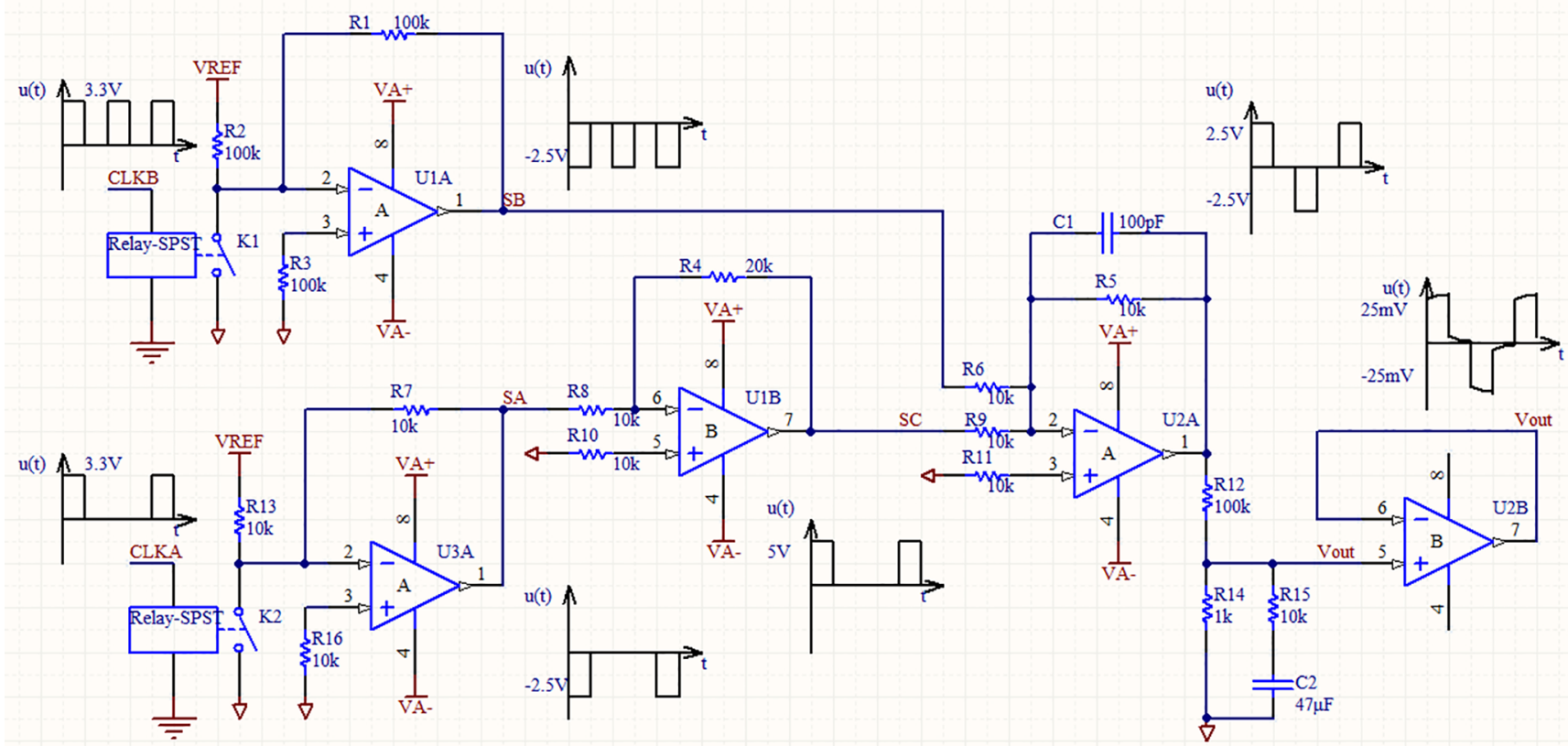

Figure 8. Schematic of the shaper circuit.

ature range of -20 to $75^{\circ} \mathrm{C}$. The power consumption of the module is $12 \mathrm{~mA}$ at $3.3 \mathrm{~V}$. For example, to generate the clock $(f=9600 \mathrm{~Hz})$ in the CSAMT mode, the MCU sets the division factor to 1280 and the CPLD divides the clock signal, which is triggered by the PPS from the GPS module. After 50 PPS counts, the MCU sets the division factor to 1600 , and the output clock frequency changes to $7680 \mathrm{~Hz}$. The above steps are then repeated, and each division is triggered by the PPS. There is a $1 \mathrm{~s}$ pause between each switch divider for the CPLD to synchronize with the MCU. The CPLD is controlled by an MCU with a serial-peripheral interface.

In the SIP mode, a clock signal with an accuracy of $\pm 30 \mathrm{ppb}$ will drift by $27 \mu \mathrm{s}$, while the entire circulation lasts $15 \mathrm{~min}$. The time drift error will cause a $21 \mathrm{mrad}$ phase error when the frequency of the transmitter is set to $128 \mathrm{~Hz}$. For high phase precision, the frequency error of the switching clock signal must be as low as possible. 


\subsection{Chopper circuit}

Figure 7 shows the schematic of the chopper circuit, which chops a high-precision DC reference voltage into a bipolar square waveform driven by the switching clock. The circuit contains voltage references, a single-pole-singlethrow (SPST) relay, and an amplifier. The voltage reference LT1019-2.5 (from Linear Technology) used is a $2.5 \mathrm{~V}$ highprecision DC reference. The relay is driven by the switching clock, which is a $3.3 \mathrm{~V}$ CMOS square waveform. The switching clock is divided from the above MCXO. The switching clock controls the connection of the $\mathrm{U} 2$ pin 3 and signal ground. The output from U2 pin1 is a bipolar square wave with an amplitude of $2.5 \mathrm{~V}$. The component U2B is an attenuator and low-pass filter, and the output decreases from $5 \mathrm{Vpp}$ to $20 \mathrm{mVpp}$ for the $E$ channel. The bandwidth is limited to $100 \mathrm{kHz}$. The amplitude of the $\mathrm{H}$ channel is $200 \mathrm{mVpp}$, which is different from the gain of the U2B.

\subsection{Shaper circuit}

A schematic of the shaper circuit is shown in Fig. 8. The shaper circuit shapes the clock output from the CPLD into a PZNZ waveform. In the shaper circuit, there are two channel clock sources (CLKA and CLKB) and one output (Vout). The CLKA and CLKB outputs are set as different frequencies such that the frequency of CLKA is double the frequency of CLKB, and the duty ratio of CLKB is $1: 3$. The width of each pulse is $2 \mathrm{~s}$. The amplifiers U1A and U3A are both used as chopper circuits, which chop the DC reference to the square waveform. The clock is transformed into a square waveform with an amplitude of $2.5 \mathrm{~V}$. The amplifier U1B is set with a gain of -2 . The amplifier U2A adds nodes SB and $\mathrm{SC}$. The waveform at node SD is a bipolar PZNZ waveform. The components R12, R14, R15, and C2 constitute an RC network to generate the PZNZ waveform based on the principles described earlier. The amplitude at the output of the shaper is approximately $50 \mathrm{mVpp}$.

\section{Test}

\subsection{MT mode}

We used the developed multifunction waveform generator to test our multifunction EM receiver (EMR6). The output of the multifunction waveform generator was connected to the input of the EMR6, which works in three sequential modes: $30 \mathrm{~min}$ for AMT mode, $24 \mathrm{~h}$ for MT mode, and $72 \mathrm{~h}$ for LMT mode. All raw data were processed using the white-noise method by the MT data processing software (SSMT2000) from Phoenix Geophysics. The results of the data processing are shown in Fig. 9. The apparent resistivity and impedance phase are present across the entire 9-decade frequency range from $1 \times 10^{-5}$ to $1 \times 10^{4} \mathrm{~Hz}$. The theoretical simulation result of the apparent resistivity was $1270 \Omega \mathrm{m}$,
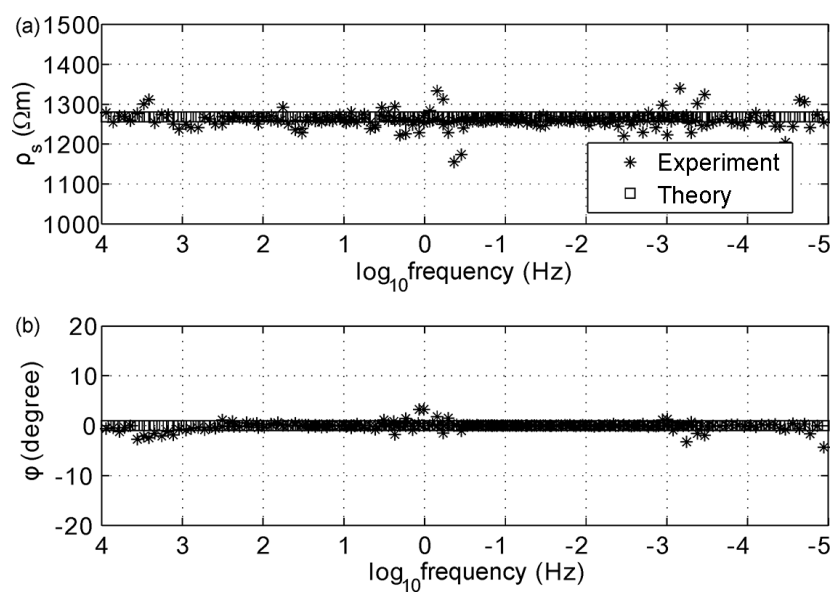

Figure 9. Apparent resistivity and impedance phase results from the EMR6 testing. (a) shows the apparent resistivity curves, and (b) shows the impedance phase curves.

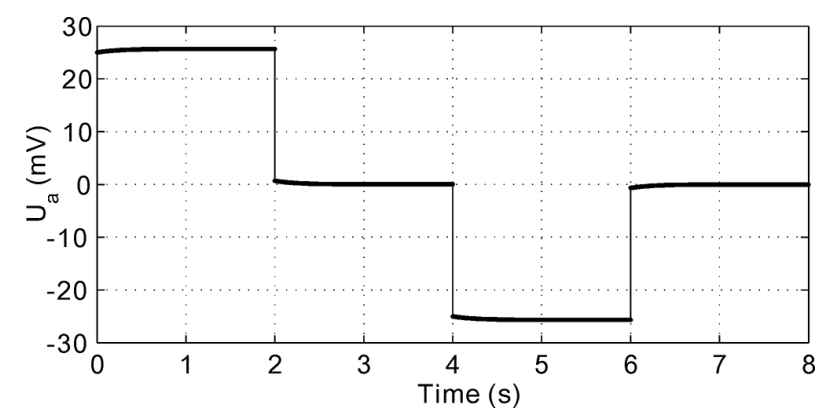

Figure 10. Time series in TDIP mode as captured by the EMR6.

and the impedance phase was approximately $0^{\circ}$. When the experimental results were compared to those from the theoretical simulation, the bias error between the experiment results and theoretical value for the apparent resistivity was $1.5 \%$.

The results of the experiment indicated that the multifunction waveform generator could test the MT receiver across the entire MT band, and the EMR6 receiver was shown to have a correct response in the target bands.

\subsection{TDIP mode}

To verify the TDIP waveform, the generator was switched to the TDIP mode and its output was connected to the EMR6 $E$ channel input; the EMR performs TDIP data acquisition during $2 \mathrm{~min}$ at a $2400 \mathrm{~Hz}$ sample rate. Figure 10 shows the time series captured by the EMR6. The full waveform was recorded for the entire time series of the $E_{1}$ channel, including the primary and secondary field waveforms. The amplitude was approximately $50 \mathrm{mVpp}$, and the "on" time and "off" time pulse widths were $2 \mathrm{~s}$.

The chargeability was calculated by dividing the secondary field into nine windows. The offset time of the first 
Table 2. Specification comparison with Agilent 33510B.

\begin{tabular}{lll}
\hline Specifications & Agilent 33510B & $\begin{array}{l}\text { Newly developed multifunction } \\
\text { waveform generator }\end{array}$ \\
\hline Function & $\begin{array}{l}\text { Sine, square, ramp, pulse, } \\
\text { triangle, PRBS, white noise }\end{array}$ & PRBS, frequency sweep, PZNZ \\
Channels & 2 & $4\left(E_{x}, E_{y}, H_{x}, H_{y}\right)$ \\
Time sync & Internal timer or Ext Trig connector & GPS \\
PRBS & $1-50 \mathrm{M}$ bps & $10 \mu \mathrm{Hz}-100 \mathrm{kHz}$ \\
Power & AC $100-240 \mathrm{~V}$ & Built-in rechargeable Li-ion battery \\
\hline
\end{tabular}

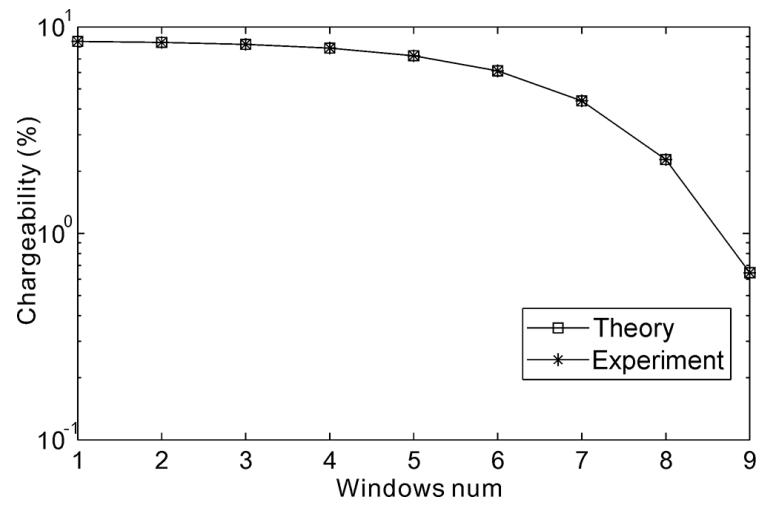

Figure 11. Comparison between the calculated results and the theoretical simulation results for TDIP mode.

window was $10 \mathrm{~ms}$; the width of the windows was $8,16,32$, $64,128,256,512,1024$, and 2048 sample points. Figure 11 shows a comparison between the measured results and those from the theoretical simulation. The chargeability was distributed from 0.7 to $9 \%$. The results show that the output from the waveform generator during the experiment response was in good agreement with the theoretical output. The bias error of the nine windows was $0.8 \%$ between the measured results from the experiment and those from the theoretical simulation.

\subsection{SIP mode}

The EMR6 and waveform generator operated in the SIP mode. To simplify the experiment, the current data of the transmitter and a variety of geometric factors were not considered. The EMR6 recorded the frequency-swept square waveform for $15 \mathrm{~min}$ and calculated the amplitude of each target frequency. Figure 12 shows the 12 target frequencies from 128 to $0.0625 \mathrm{~Hz}$. The amplitudes measured in the experiment were approximately $12.74 \mathrm{mV}$, and the phases were approximately $0^{\circ}$. The bias error between the experiment and theory was $0.5 \%$ across the entire frequency range.
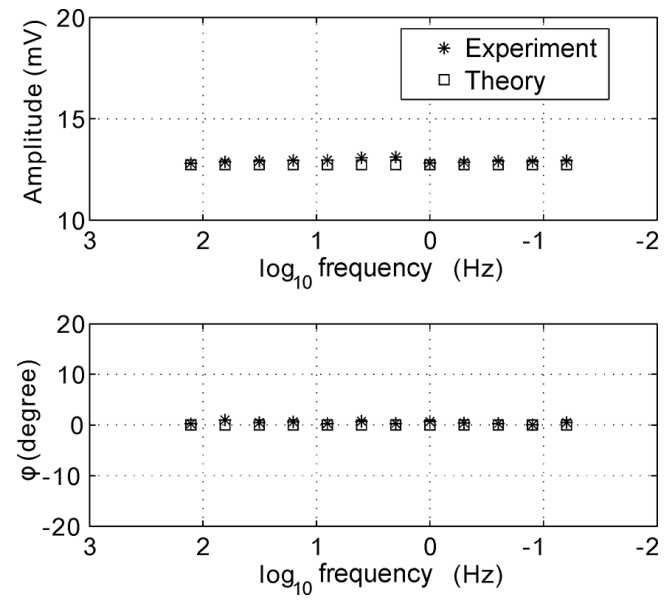

Figure 12. Comparison between the experimental results and the theoretical simulation results for the SIP mode.
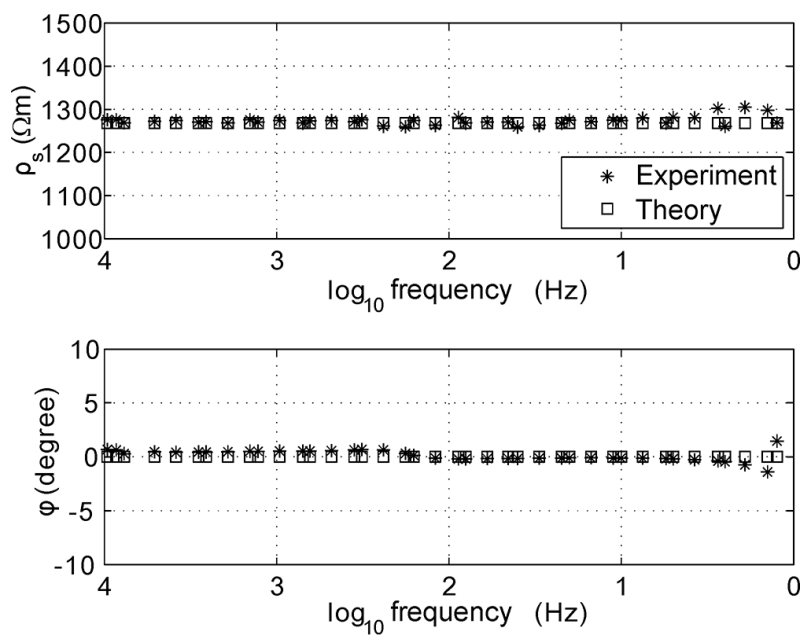

Figure 13. Comparison between the calculated results and the simulation results for the CSAMT mode.

\subsection{CSAMT mode}

The EMR6 and waveform generator operated in the CSAMT mode and recorded the $E$ - and $H$-channel swept square waveforms. The Cagniard apparent resistivity and impedance 
phase were calculated. The receiver recorded the swept frequency waveform for $50 \mathrm{~min}$. Figure 13 shows the results calculated for the 41 target frequency points from 9600 to $0.9375 \mathrm{~Hz}$. The apparent resistivity measured in the experiment was approximately $1268 \Omega \mathrm{m}$, and the phase was approximately $0^{\circ}$. The bias error between the experiment and theory was $1.3 \%$ across the entire frequency range.

\section{Discussion}

We developed a special multifunction waveform generator to meet the testing requirements of the EMR6 receiver. The waveform generator supports a broadband, low-noise PRBS for MT, repeating frequency sweep square waveform for CSAMT and SIP with a programmable frequency step list, and PZNZ waveform containing both primary and secondary fields for the TDIP mode. Moreover, additional requirements for the waveform generator included (1) ease of use, autorepeat circulation of output according to the scheduled frequency step list; (2) high-precision phase and time synchronization; and (3) low power consumption for the LMT mode test, which has the capacity of the built in Li-ion battery that allows for 1 week of operation.

Based on the results of the above experiment, Table 2 presents the comparison of the specifications of the developed multifunction waveform generator and the Agilent 33510B function waveform generator. The presented results indicate that Agilent 33510B is not suitable for EM receiver testing. The developed multifunction waveform generator is a better signal source for this purpose.

Total harmonic distortion (THD) is another important factor determining the performance of the EM receiver. We have used a low-distortion signal generator (DS360) from Stanford Research Systems as a pure signal source. This function is not contained in the current work, and it may be realized in a future development.

\section{Conclusions}

The performance of the existing commercial function/arbitrary waveform generator is deficient with regard to time synchronization and waveform requirements. The multifunction waveform generator described in this paper was found to be useful for conducting EM receiver testing for multiple EM methods. The results of the testing show that the multifunction waveform generator could provide three mode signals containing independent broadband signals with different spectral characteristics, white noise, a repeating swept square waveform, and a PZNZ waveform consisting of primary and secondary fields. The apparent resistivity and impedance phase of the broadband white-noise source was very flat across a wide frequency band. The theoretical design of the TDIP waveform was shown to have a correct response. In the SIP and CSAMT modes, the results of the experiment and theoretical simulation were a close match. In addition, the generator had other advantages in that it was easy to use and had low power consumption.

Furthermore, various parameters, such as the programmable frequency step schedule in the CSAMT and SIP modes and the pulse width of the TDIP waveform, are configurable by the user through the hardware interface or by loading the memory.

Data availability. The circuit schematics of the multifunction waveform generator are available upon request (ck@cugb.edu.cn).

Author contributions. KC developed the required hardware and software. SJ created the overall design and performed the tests. MD was the chopper and shaper circuit technology consultant.

Competing interests. The authors declare that they have no conflict of interest.

Acknowledgements. General funding was provided by the National High Technology Research and Development Program of China (2014AA06A603), National Science Foundation of China (61531001), Central University Fundamental Research Project of the Ministry of Education (2652015403), and Key Development Program of China (2016YFC0303100). We are thankful for the data processing software from Phoenix Geophysics. We would also like to thank Editage (www.editage.cn) for their English language editing services.

Edited by: Mark Paton

Reviewed by: Rujun Chen and one anonymous referee

\section{References}

Amrani, M. E. H., Dowdeswell, R. M., Payne, P. A., and Persaud, K. C.: Pseudo-random binary sequence interrogation technique for gas sensors, Sensor. Actuat. B-Chem., 47, 118-124, 1998.

Cagniard, L.: Basic theory of the magneto-telluric method of geophysical prospecting, Geophysics, 18, 605-635, 1953.

Chen, R. J., Yao, H. C., and Liu, S. L.: Automatic testing system of pseudo-random multi-frequency instrument receiver, Ref. No: CN200910044457, Chinese Patent, 2010.

Ge, S. C., Deng, M., Chen, K., and Shi, X. Y.: Broadband signal generator for the approximation of a magnetotelluric source for indoor testing, J. Geophys. Eng., 13, 612-621, 2016.

He, Z. X., Chen, R. J., Liu, X. J. L., and He, L. F.: Magnetotelluric instrument performance evaluation method, Ref. No: CN200910237810, Chinese Patent, 2011.

Johnson, I. M.: Spectral induced polarization parameters as determined through time-domain measurements, Geophysics, 49, 1993-2003, 1984.

Key, K. W.: Application of broadband marine magnetotelluric exploration to a three-dimensional salt structure and a fast- 
spreading ridge, $\mathrm{PhD}$ thesis, University of California, San Diego, 97-105, 2003.

KMS: KMS-820, http://www.kmstechnologies.com/kms_ company_overview.html, last access: 1 January 2017.

Marshall, D. J. and Madden, T. R.: Induced Polarization, a study of its causes, Geophysics, 24, 790-816, 1959.

Metronix: ADU-07e, http://www.metronix.de/metronix/index.php, last access: 1 January 2017.

Osinowo, O. O. and Olayinka, A. I.: Very low frequency electromagnetic (VLF-EM) and electrical resistivity (ER) investigation for groundwater potential evaluation in a complex geological terrain around the Ijebu-Ode transition zone, southwestern Nigeria, J. Geophys. Eng., 9, 374-396, 2012.

Phoenix Geophysics: V8 receiver, http://www.phoenix-geophysics. com/products/receivers/v8/, last access: 1 January 2017.
Sandberg, S. K. and Hohmann, G. W.: Controlled-source audiomagnetotellurics in geothermal exploration, Geophysics 47, 100116, 1982.

Scheuermann, A.: Electric and electromagnetic measurement methods in civil and environmental engineering, J. Geophys. Eng., 13, E1-E2, 2016.

Wei, W., Jin, S., Ye, G., Deng, M., Jing, J., Unsworth, M., and Jones, A. G.: Conductivity structure and rheological property of lithosphere in Southern Tibet inferred from super-broadband magnetotelluric sounding, Sci. China Earth Sci., 53, 189-202, 2010.

Zonge: GDP32, http://zonge.com/instruments-home/instruments/ receivers/, last access: 1 January 2017. 